\title{
Improved zero-sum distinguisher for full round Keccak-f permutation
}

\author{
DUAN Ming $^{1,2^{*}} \&$ LAI XueJia ${ }^{1}$ \\ ${ }^{1}$ Department of Computer Science and Engineering, Shanghai Jiao Tong University, Shanghai 200240, China; \\ ${ }^{2}$ Basic Courses Department, University of Foreign Language, Luoyang 471003, China
}

Received August 2, 2011; accepted September 30, 2011

\begin{abstract}
Keccak is one of the five hash functions selected for the final round of the SHA-3 competition, and its inner primitive is a permutation called Keccak-f. In this paper, we observe that for the inverse of the only nonlinear transformation in Keccak-f, the algebraic degree of any output coordinate and the one of the product of any two output coordinates are both 3, which is 2 less than its size of 5. Combining this observation with a proposition on the upper bound of the degree of iterated permutations, we improve the zero-sum distinguisher for the Keccak- $f$ permutation with full 24 rounds by lowering the size of the zero-sum partition from $2^{1590}$ to $2^{1575}$.
\end{abstract}

hash functions, higher order differentials, algebraic degree, zero-sum, SHA-3

Citation: Duan M, Lai X J. Improved zero-sum distinguisher for full round Keccak-f permutation. Chin Sci Bull, 2012, 57: 694-697, doi: 10.1007/s11434011-4909-x

Zero-sum distinguishers, introduced by Aumasson and Meier and presented at the rump session of CHES 2009, are a method for generating zero-sum structures for iterated permutations, which combine higher order differential technique with inside-out technique and are mainly decided by the algebraic degree of the permutation. In the public comment on the NIST Hash competition 2010, zero-sum distinguishers are shown to be deterministic and valid, although they generate zero-sum structures with only a small advantage over the generic method. Zero-sum distinguishers can also be used to create partitions of inputs into many different zero-sum structures for the permutation [1].

Keccak is a family of cryptographic sponge functions and is one of the five hash functions selected for the third (and final) round of the SHA-3 competition. Its core component is a permutation named Keccak-f, which is composed of several iterations of five transformations. A first zero-sum distinguisher for the Keccak-f permutation with 16 rounds was given in 2009. Since then, zero-sum distinguishers for

*Corresponding author (email: dodoxixi@gmail.com) the Keccak- $f$ permutation with a greater number of rounds were obtained [1,2], with the smallest known zero-sum partition for the Keccak- $f$ permutation with full 24 rounds having size $2^{1590}$.

In this paper, we study the properties of the inverse of the nonlinear transformation in Keccak-f, and observe that the algebraic degree of the product of any two output coordinates of this inverse is 2 less than its size. This enables us construct a zero-sum partition for the Keccak- $f$ permutation with full 24 rounds of size $2^{1575}$.

\section{Zero-sum distinguishers}

We first introduce the notions of higher order derivatives related to zero-sum distinguishers.

\subsection{Higher order derivatives}

Higher order derivatives were introduced into cryptography by Lai in [3]. 
Definition 1. Let $f(x)$ be a Boolean function from $F_{2}^{n}$ to $F_{2}$. The derivative of $f$ at point $a \in F_{2}^{n}$ is defined by

$$
\Delta_{a} f(x)=f(x+a)+f(x) .
$$

The $i$-th $(i>1)$ derivative of the function $f$ at points $\left\{a_{1}\right.$, $\left.a_{2, \ldots,} a_{i}\right\}$ is defined by

$$
\Delta_{a_{1}, \ldots, a_{i}}^{(i)} f(x)=\Delta_{a_{i}}\left(\Delta_{a_{1}, \ldots, a_{i-1}}^{(i-1)} f(x)\right),
$$

where $\Delta_{a_{1}, \ldots, a_{i-1}}^{(i-1)} f(x)$ is the $(i-1)$-th derivative of $f$ at points $\left\{a_{1}, a_{2}, \ldots, a_{i-1}\right\}$. The 0 -th derivative of $f$ is defined to be $f(x)$ itself.

Higher order derivatives should be computed at points that are linearly independent, otherwise the derivative will trivially be zero. Note that the degree of the derivative of a function is at least 1 less than the degree of the function. This implies that the $(d+1)$-th derivative of an $n$-variable Boolean function of degree $d$ is zero, and this is used in many cryptanalysis methods including zero-sum distinguishers.

\subsection{Zero-sum properties}

Note that the permutation used in a hash function does not depend on a secret parameter, and this property of the permutation can be exploited from the middle. The zero-sum property introduced by Aumasson and Meier is based on higher order differential technique and inside-out technique. The main idea is to take higher order derivatives at initial states inverted from an intermediate internal state subspace, which differs from traditional higher order differential distinguishers that take derivatives directly from the initial state subspace. So zero-sum distinguishers lower the degree of higher order derivatives by nearly half with the added cost of some inverted computations.

We now give the definitions of zero-sum and zero-sum partitions. Further details can be found in [1].

Definition 2. Let $F$ be a function from $F_{2}^{n}$ into $F_{2}^{m}$. A zero-sum for $F$ of size $K$ is a subset $\left\{x_{1}, x_{2}, \ldots, x_{K}\right\} \subset F_{2}^{n}$ of elements which sum to zero and for which the corresponding images by $F$ also sum to zero. That is,

$$
\sum_{i=1}^{K} x_{i}=\sum_{i=1}^{K} F\left(x_{i}\right)=0 .
$$

Definition 3. Let $P$ be a permutation from $F_{2}^{n}$ into $F_{2}^{n}$. A zero-sum partition for $F$ of size $K=2^{k}$ is a collection of $2^{n-k}$ disjoint zero-sums $X_{i}=\left\{x_{i, 1}, x_{i, 1, \ldots, x_{i, 2^{k}}}\right\} \subset F_{2}^{n}$. That is,

$$
\bigcup_{i=1}^{2^{n-k}} X_{i}=F_{2}^{n} \text { and } \sum_{j=1}^{2^{k}} x_{i, j}=\sum_{j=1}^{2^{k}} P\left(x_{i, j}\right)=0, \forall 1 \leqslant i \leqslant 2^{n-k} \text {. }
$$

\section{Description of the Keccak-f permutation}

The size of Keccak-f is 1600, and the state can be represented by a 3 -dimensional binary matrix of size $5 \times 5 \times 64$. The five transformations are respectively called $\theta, \rho, \pi, l$ and $\chi$. Only the transformation $\chi$ is nonlinear, its degree being 2 while the degree of its inverse is 3 . The Boolean components of $\chi$ are listed in Table 1. More details of the Keccak-f permutation are available in the website of the NIST Hash competition.

\section{Generalized and intuitive upper bound of the degree of iterated permutations}

High algebraic degree is an important design principle for cryptographic algorithms. It is difficult to determine the algebraic degree when the number of rounds in the algorithm is too big. Estimating the upper bound on the algebraic degree is relatively feasible. In [4], Canteaut and Videau gave an upper bound on the degree of composition of nonlinear functions and used it to estimate the algebraic degree of the whole algorithm. In the rump session of Crypto 2010, Boura et al. [2] proposed an improved upper bound for iterated permutations with a nonlinear layer composed of parallel applications of small balanced S-boxes. We next discuss this latter upper bound and give a proposition for a visualized bound in some cases.

Theorem 1[2]. Let $f$ be a function from $F_{2}^{n}$ into $F_{2}^{n}$ corresponding to the concatenation of $m$ smaller balanced S-boxes, $S_{1}, \ldots, S_{m}$, defined over $F_{2}^{n}$. Let $\delta_{k}$ be the maximal degree of the product of any $k$ coordinates from any one of these smaller S-boxes. Then, for any function $G$ from $F_{2}^{n}$ into $F_{2}^{l}$, we have

$$
\operatorname{deg}(G \circ F) \leqslant n-\frac{n-\operatorname{deg}(G)}{\gamma},
$$

where

$$
\gamma=\max _{1 \leqslant i \leqslant n_{0}-1} \frac{n_{0}-i}{n_{0}-\delta_{i}}
$$

Most notably, we have

Table 1 Boolean components of $\chi$

\begin{tabular}{cc}
\hline Output & Corresponding Boolean function \\
\hline$\chi_{0}$ & $x_{0}+x_{2}+x_{1} x_{2}$ \\
$\chi_{1}$ & $x_{1}+x_{3}+x_{2} x_{3}$ \\
$\chi_{2}$ & $x_{2}+x_{4}+x_{3} x_{4}$ \\
$\chi_{3}$ & $x_{0}+x_{3}+x_{0} x_{4}$ \\
$\chi_{4}$ & $x_{1}+x_{4}+x_{0} x_{1}$ \\
\hline
\end{tabular}




$$
\operatorname{deg}(G \circ F) \leqslant n-\frac{n-\operatorname{deg}(G)}{n_{0}-1} .
$$

Moreover, if $n_{0} \geqslant 3$ and all S-boxes have degree at most $n_{0}-2$, we have

$$
\operatorname{deg}(G \circ F) \leqslant n-\frac{n-\operatorname{deg}(G)}{n_{0}-2} .
$$

Lemma 1. Let $f$ be a function from $F_{2}^{n}$ into $F_{2}^{n}$ corresponding to the concatenation of $m$ smaller S-boxes, $S_{1}, \ldots$, $S_{m}$, defined over $F_{2}^{n_{0}}$. Let $\delta_{k}$ be the maximal degree of the product of any $k$ coordinates of any one of these smaller S-boxes. If $n_{0} \geqslant 2 k-1(k \geqslant 1)$ and $\delta_{i} \leqslant n_{0}-1$ for any $i$ from 1 to $n_{0}-1$, and $\delta_{i} \leqslant n_{0}-2$ for any $i$ from 1 to $k-1(k \geqslant 2)$, then

$$
\left(n_{0}-k\right)\left(n_{0}-\delta_{i}\right)-\left(n_{0}-i\right) \geqslant 0
$$

for any $i$ from 1 to $n_{0}-1$.

Proof: When $k=1$, then we have

$$
\begin{aligned}
\left(n_{0}-k\right)\left(n_{0}-\delta_{i}\right)-\left(n_{0}-i\right) & =\left(n_{0}-1\right)\left(n_{0}-\delta_{i}\right)-\left(n_{0}-i\right) \\
& \geqslant\left(n_{0}-1\right)-\left(n_{0}-i\right) \\
& \geqslant\left(n_{0}-1\right)-\left(n_{0}-1\right)=0 .
\end{aligned}
$$

When $k \geqslant 2$, then we have

$$
\begin{aligned}
\left(n_{0}-k\right)\left(n_{0}-\delta_{i}\right)-\left(n_{0}-i\right) & \geqslant 2\left(n_{0}-k\right)-\left(n_{0}-i\right) \\
& \geqslant 2\left(n_{0}-k\right)-\left(n_{0}-1\right) \\
& \geqslant n_{0}-2 k+1 \geqslant 0 .
\end{aligned}
$$

First, from Theorem 1, we know that the condition that the S-boxes are balanced in confirms that the inequality $\delta_{i} \leqslant n_{0}-1$ is satisfied for any $i$ from 1 to $n_{0}-1$. This is not a necessary condition, however, and it is not necessary to limit the condition to balanced S-boxes. That is, the condition in Theorem 1 can be generalized.

Second, note that the parameter $\gamma$ in the theorem is the maximum value of $\frac{n_{0}-i}{n_{0}-\delta_{i}}$ for $i$ from 1 to $n_{0}-1$. Lemma 1 tells us that the positive integer $n_{0}-k$ also suffices in some cases. That is, the inequality $n_{0}-k \geqslant \frac{n_{0}-i}{n_{0}-\delta_{i}}$ always holds under the conditions of the lemma, so we get $\gamma \leqslant\left(n_{0}-k\right)$.

With the more generalized condition and more determinate parameter, we have the following intuitive upper bound by combining the theorem and the lemma.

Proposition 1. Let $f$ be a function from $F_{2}^{n}$ into $F_{2}^{n}$ corresponding to the concatenation of $m$ smaller S-boxes, $S_{1}, \ldots, S_{m}$, defined over $F_{2}^{n_{0}}$. Let $\delta_{k}$ be the maximal degree of the product of any $k$ coordinates of any one of these smaller S-boxes. If $n_{0} \geqslant 2 k-1(k \geqslant 1)$ and $\delta_{i} \leqslant n_{0}-1$ for any $i$ from 1 to $n_{0}-1$, and $\delta_{i} \leqslant n_{0}-2$ for any $i$ from 1 to $k-1$ $(k \geqslant 2)$, then, for any function $G$ from $F_{2}^{n}$ into $F_{2}^{l}$, we have

$$
\operatorname{deg}(G \circ F) \leqslant n-\frac{n-\operatorname{deg}(G)}{n_{0}-k} .
$$

Actually, when the conditions of Proposition 1 are satisfied and $n_{0}$ is an even number, then the parameter $\gamma$ can be improved to $n_{0}-k-1 / 2$, but a discussion of this is not relevant to this paper.

\section{Improved zero-sum distinguisher for Keccak-f}

\subsection{An observation about Keccak- $f$}

We give the Boolean components of $\chi^{-1}$ and the product of any two output coordinates of the transformation in Tables 2 and 3 respectively.

From Table 3, an interesting observation about the inverse of the nonlinear layer of Keccak- $f$ can be obtained.

Observation: For the inverse of the only nonlinear transformation in Keccak- $f$, the algebraic degree of any output coordinate and the one of the product of any two output coordinates are both 3 , which is 2 less than its size of 5 .

\subsection{Improved zero-sum partition for full 24-rounds Keccak- $f$ permutation}

Let $R$ denote the Keccak- $f$ round permutation. Note that $\chi$

Table 2 Boolean components of $\chi^{-1}$

\begin{tabular}{cc}
\hline Output & Corresponding Boolean function \\
\hline$\chi_{0}^{-1}$ & $x_{0}+x_{2}+x_{4}+x_{1} x_{2}+x_{1} x_{4}+x_{3} x_{4}+x_{1} x_{3} x_{4}$ \\
$\chi_{1}^{-1}$ & $x_{0}+x_{1}+x_{3}+x_{0} x_{2}+x_{0} x_{4}+x_{2} x_{3}+x_{0} x_{2} x_{4}$ \\
$\chi_{2}^{-1}$ & $x_{1}+x_{2}+x_{4}+x_{0} x_{1}+x_{1} x_{3}+x_{3} x_{4}+x_{0} x_{1} x_{3}$ \\
$\chi_{3}^{-1}$ & $x_{0}+x_{2}+x_{3}+x_{0} x_{4}+x_{1} x_{2}+x_{2} x_{4}+x_{1} x_{2} x_{4}$ \\
$\chi_{4}^{-1}$ & $x_{1}+x_{3}+x_{4}+x_{0} x_{1}+x_{0} x_{3}+x_{2} x_{3}+x_{0} x_{2} x_{3}$ \\
\hline
\end{tabular}

Table 3 Product of any two output coordinates of $\chi^{-1}$

\begin{tabular}{ll}
\hline Output & \multicolumn{1}{c}{ Corresponding Boolean function } \\
\hline$\chi_{0}^{-1} \chi_{1}^{-1}$ & $x_{0}+x_{0} x_{1}+x_{0} x_{2}+x_{0} x_{3}+x_{0} x_{4}+x_{0} x_{2} x_{3}+x_{0} x_{2} x_{4}$ \\
$\chi_{0}^{-1} \chi_{2}^{-1}$ & $x_{2}+x_{4}+x_{0} x_{2}+x_{0} x_{4}+x_{1} x_{2}+x_{1} x_{4}+x_{3} x_{4}+x_{0} x_{3} x_{4}+x_{1} x_{3} x_{4}$ \\
$\chi_{0}^{-1} \chi_{3}^{-1}$ & $x_{0}+x_{2}+x_{0} x_{3}+x_{0} x_{4}+x_{1} x_{2}+x_{2} x_{3}+x_{2} x_{4}+x_{3} x_{4}+x_{1} x_{2} x_{3}+x_{1} x_{2} x_{4}$ \\
$\chi_{0}^{-1} \chi_{4}^{-1}$ & $x_{4}+x_{0} x_{3}+x_{0} x_{4}+x_{1} x_{4}+x_{2} x_{4}+x_{1} x_{2} x_{4}+x_{1} x_{3} x_{4}$ \\
$\chi_{1}^{-1} \chi_{2}^{-1}$ & $x_{1}+x_{0} x_{1}+x_{1} x_{2}+x_{1} x_{3}+x_{1} x_{4}+x_{0} x_{1} x_{3}+x_{1} x_{3} x_{4}$ \\
$\chi_{1}^{-1} \chi_{3}^{-1}$ & $x_{0}+x_{3}+x_{0} x_{1}+x_{0} x_{2}+x_{0} x_{4}+x_{1} x_{3}+x_{2} x_{3}+x_{0} x_{1} x_{4}+x_{0} x_{2} x_{4}$ \\
$\chi_{1}^{-1} \chi_{4}^{-1}$ & $x_{1}+x_{3}+x_{0} x_{1}+x_{0} x_{3}+x_{1} x_{4}+x_{2} x_{3}+x_{3} x_{4}+x_{0} x_{2} x_{3}+x_{2} x_{3} x_{4}$ \\
$\chi_{2}^{-1} \chi_{3}^{-1}$ & $x_{2}+x_{0} x_{2}+x_{1} x_{2}+x_{2} x_{3}+x_{2} x_{4}+x_{0} x_{2} x_{4}+x_{1} x_{2} x_{4}$ \\
$\chi_{2}^{-1} \chi_{4}^{-1}$ & $x_{1}+x_{4}+x_{0} x_{1}+x_{1} x_{2}+x_{1} x_{3}+x_{2} x_{4}+x_{3} x_{4}+x_{0} x_{1} x_{2}+x_{0} x_{1} x_{3}+x_{0} x_{3} x_{4}+x_{2} x_{3} x_{4}$ \\
$\chi_{3}^{-1} \chi_{4}^{-1}$ & $x_{3}+x_{0} x_{3}+x_{1} x_{3}+x_{2} x_{3}+x_{3} x_{4}+x_{0} x_{1} x_{3}+x_{0} x_{2} x_{3}$ \\
\hline
\end{tabular}


is the only nonlinear transformation in $R$. Combining our earlier observation and Proposition 1 , we have

$$
\operatorname{deg}(G \circ R)=\operatorname{deg}(G \circ \chi) \leqslant n-\frac{n-\operatorname{deg}(G)}{3}
$$

and

$$
\operatorname{deg}\left(G \circ R^{-1}\right)=\operatorname{deg}\left(G \circ \chi^{-1}\right) \leqslant n-\frac{n-\operatorname{deg}(G)}{2},
$$

where $G$ is any function from $F_{2}^{5}$ into $F_{2}^{l}$. Our upper bounds on the degree of the inverse of Keccak-f are less than the bounds in [2] when the number of rounds is more than seven. The comparisons are listed in Table 4.

Combining these upper bounds on $\operatorname{deg}\left(R^{\gamma}\right)$ with those in [2] and our lowered upper bounds on $\operatorname{deg}\left(R^{-\gamma}\right)$, we have a zero-sum partition of size $2^{1575}$ for the full Keccak- $f$ permutation. This is smaller than the original size of $2^{1590}$, as

Table 4 Comparison of the upper bounds on $\operatorname{deg}\left(R^{-\gamma}\right)$

\begin{tabular}{ccc}
\hline Round & Bound in [2] & Our bound \\
\hline 1 & 3 & 3 \\
2 & 9 & 9 \\
3 & 27 & 27 \\
4 & 81 & 81 \\
5 & 243 & 243 \\
6 & 729 & 729 \\
7 & 1309 & 1164 \\
8 & 1503 & 1382 \\
9 & 1567 & 1491 \\
10 & 1589 & 1545 \\
11 & 1596 & 1572 \\
12 & 1598 & 1586 \\
13 & 1599 & 1593 \\
14 & 1599 & 1596 \\
15 & 1599 & 1598 \\
16 & 1599 & 1599 \\
\hline
\end{tabular}

confirmed in the updated version of [2] appearing in the Preproceedings of FSE 2011. Indeed, one can consider the intermediate states after the three linear layers $\theta, \rho$ and $\pi$, in the 12-th round of Keccak- $f$ in any subspace $V$ corresponding to a collection of 315 rows, because the upper bound of the backward 11 rounds is 1572 and that of the forward 12 rounds is 1536 [2].

\section{Discussion}

In this paper, we lower the size of a zero-sum partition for the Keccak-f permutation with full 24 rounds based on an interesting observation about the inverse of the nonlinear transformation in the permutation. One can verify that some of the products of three output coordinates also have a degree of only 3 . This property may be used for more practical cryptanalysis of Keccak in the future.

The authors express their great thanks to $C$. Boura and the anonymous reviewers for their helpful comments. This work was supported by the National Natural Science Foundation of China (60573032, 60773092 and 61073149), and Research Fund for the Doctoral Program of Higher Education of China (20090073110027).

1 Boura C, Canteaut A. Zero-sum distinguishers for iterated permutations and application to Keccak- $f$ and Hamsi-256. In: Proceedings of the 17th International Workshop on Selected Areas in Cryptography 2010 Aug 12-13, Waterloo, Ontario, Canada. Waterloo: LNCS Springer Press, 2010. 1-17

2 Boura C, Canteaut A, Cannière C D. Higher-order differential properties of Keccak and Luffa, In: Proceedings of the 18th International Workshop on Fast Software Encryption 2011 Feb 14-16, Lyngby, Denmark. Lyngby: LNCS Springer Press, 2011. 252-269

3 Lai X J. Higher order derivatives and differential cryptanalysis. In: Communications and Cryptography: Two Sides of One Tapestry 1994, Switzerland. Switzerland: Kluwer Adademic Publishers, 1994. 227-233

4 Canteaut A,Videau M. Degree of composition of highly nonlinear functions and applications to higher order differential cryptanalysis. In: Advances in Cryptology-EUROCRYPT 2002, International Conference on the Theory and Applications of Cryptographic Techniques, Apr 28-May 2, Amsterdam, The Netherlands. Amsterdam: LNCS Springer Press, 2002. 518-533

Open Access This article is distributed under the terms of the Creative Commons Attribution License which permits any use, distribution, and reproduction in any medium, provided the original author(s) and source are credited. 